\title{
Ethnobotanique des plantes médicinales chez les bergers peuls de Widou Thiengoly de la commune de Téssékéré (Ferlo-Nord Sénégal).
}

CISSE Ababacar', GUEYE Mathieu', KA Abdou², NDIAYE François ${ }^{1}$, KOMA Soulèye ${ }^{1}$, AKPO Léonard Elie $^{3}$

1'Institut Fondamental d'Afrique Noire (IFAN), Département de Botanique et Géologie, Laboratoire de Botanique, BP 206 Dakar, Sénégal; ababacarcisse4baye@gmail.com

2Unité Mixte Internationale (UCAD- CNRS-UGB-USTTB-CNRST) BP 5005, Dakar Sénégal

3Université Cheikh Anta Diop de Dakar, Faculté des Sciences et Techniques, Laboratoire d'Écologie, BP 5005 , Dakar (Sénégal).

Original submitted in on $16^{\text {th }}$ December 2015. Published online at www.m.elewa.org on 29th February 2016 http://dx.doi.org/10.4314/jab.v98i1.6

\section{RÉSUMÉ}

Objectif: Le savoir indigène à Widou Thiengoly est entre les mains des bergers qui vivent dans des écosystèmes souvent fragilisés. II est normal de préserver ce savoir nomade et ancestral.

Méthodologie et résultats : Ainsi, nous avons mené des enquêtes auprès des bergers peuls de Widou. Un guide d'entretien a été utilisé pour recueillir les informations. Nous avons inventorié 74 espèces réparties dans 32 familles botaniques. Les familles les plus représentées sont les Leguminoseae $(27 \%)$ et les Combretaceae (10\%). Soixante quinze pathologies sont traitées par ces espèces médicinales. Les organes les plus utilisés sont les feuilles et les racines. Ces espèces médicinales et ces organes sont souvent utilisés en association dans le traitement des pathologies.

Conclusion et application des résultats : Les ressources végétales jouent un rôle important dans la santé des populations surtout rurales et pose en même temps la nécessité de la prise en compte des connaissances ancestrales et traditionnelles dans les politiques de santé publique notamment en Afrique et surtout en zone rurale.

Mots clés : Indigène, Widou Thiengoly, bergers, nomade, espèces médicinales, pathologies, santé publique, zone rurale

Ethnobotanical medicinal plants among Fulani herders Widou Thiengoly the common Tessekere (North Ferlo Senegal).

ABSTRACT

Objective: Indigenous knowledge in Widou Thiengoly is in the hands of the herders who live in in this fragile ecosystem. It is normal to preserve this native and ancestral knowledge.

Methodology and results: Therefore, a survey was conducted among the Widou Fulani herdsmen. An interview guide was used to collect the information. An inventory of 74 species divided in 32 botanical families was made. The most representative families were the Leguminoseae with $27 \%$ and the combretaceae with $10 \%$. Seventy five diseases were treated by these medicinal species. The most used organs were the leaves and the roots. These medicinal species and these organs are often used in combination to treat these diseases. 

Thiengoly de la commune de Téssékéré (Ferlo-Nord Sénégal).

Conclusion and application of results: The vegetable resources play an important role in the health of populations especially rural and pose the same time the necessity of the consideration of the ancestral and traditional knowledge in public health policies in particular in Africa and especially in rural area.

Key words: Indigenous, Widou Thiengoly, herders, native, medicinal species, diseases, public health, rural area

\section{INTRODUCTION}

Les végétaux ont toujours profité et profitent encore aux populations rurales détentrices d'un patrimoine inestimable et qui bénéficient d'une grande variabilité de ressources biologiques utiles et bien souvent indispensables à leur survie: médicinale, alimentaire, bois d'œuvre et de feu, perches (Gueye et al. 2012). Les plantes médicinales jouent un rôle important dans les problèmes de santé publique. En effet, $80 \%$ pour cent de la population mondiale dépend principalement de la médecine traditionnelle pour le traitement des maux (Cunningham, 1993). L'Organisation Mondiale de la Santé (OMS) estime que $80 \%$ de la population des pays en développement sont tributaires de la médecine traditionnelle, en recourant principalement aux extraits des plantes pour satisfaire leurs besoins de soins de santé. En effet, en Afrique, les guérisseurs traditionnels ne disposant que des ressources naturelles comme seul arsenal thérapeutique, soignent dans certains cas plus de $90 \%$ de la population (Sofowora, 1982). Les plantes

\section{MATERIEL ET METHODES}

La zone d'étude : Cette étude a été menée à Widou Thiengoly, situé dans la région de Louga, département de Linguère, Sous-préfecture de Yang Yang, Commune de Téssékéré (Figure1) dont la population est dominée par les Peuls. L'accès difficile aux centres de santé nous a poussés à travailler dans cette zone.

La collecte des données : La technique des entretiens ouverts semi-structurés a été utilisée. Les conversations occasionnelles et les observations directes ont été exploitées. Ces outils participatifs permettent d'estimer les connaissances et de solliciter les réponses (Martin, 1995). Leur caractère souple et informel a permis la médicinales demeurent encore une source de soins médicaux dans les pays en voie de développement, en l'absence d'un système médical moderne (Tabuti et al. 2003). Ce savoir indigène est principalement entre les mains des collectivités locales qui vivent dans des écosystèmes souvent fragilisés. Ainsi, au rythme de dégradation actuelle des forêts, il paraît nécessaire de procéder à la collecte de ces informations et à la préservation de ce patrimoine. En effet, la prise en compte du savoir paysan dans tout programme de recherche développement est admise aujourd'hui comme une nécessité. D'ailleurs, plusieurs auteurs, dont Kilahama (1998) ont admis que les connaissances autochtones sont un outil indispensable aux différentes stratégies de vulgarisation mais aussi de sauvegarde du savoir traditionnel.C'est dans ce cadre que nous nous sommes intéressés à la diversité des espèces utilisées par les bergers peuls de Widou Thiengoly de la commune de Téssékéré (Sénégal).

participation active et détendue des différentes personnes rencontrées. Les thèmes abordés ont essentiellement trait à la diversité des ressources, leur préparation, leur administration et leur nom en Peul. Lors de nos enquêtes, nous avons toujours sollicité d'une part l'aide d'un guide interprète qui comprend la langue du terroir et qui connaît bien les espèces, et d'autre part, une sortie de terrain afin de voir si possible les espèces citées et récolter des échantillons d'herbiers. Les sorties de terrain ont été le plus souvent effectuées en compagnie de l'informateur qui nous montrait directement les espèces indiquées contre les pathologies au cours d'une promenade. 


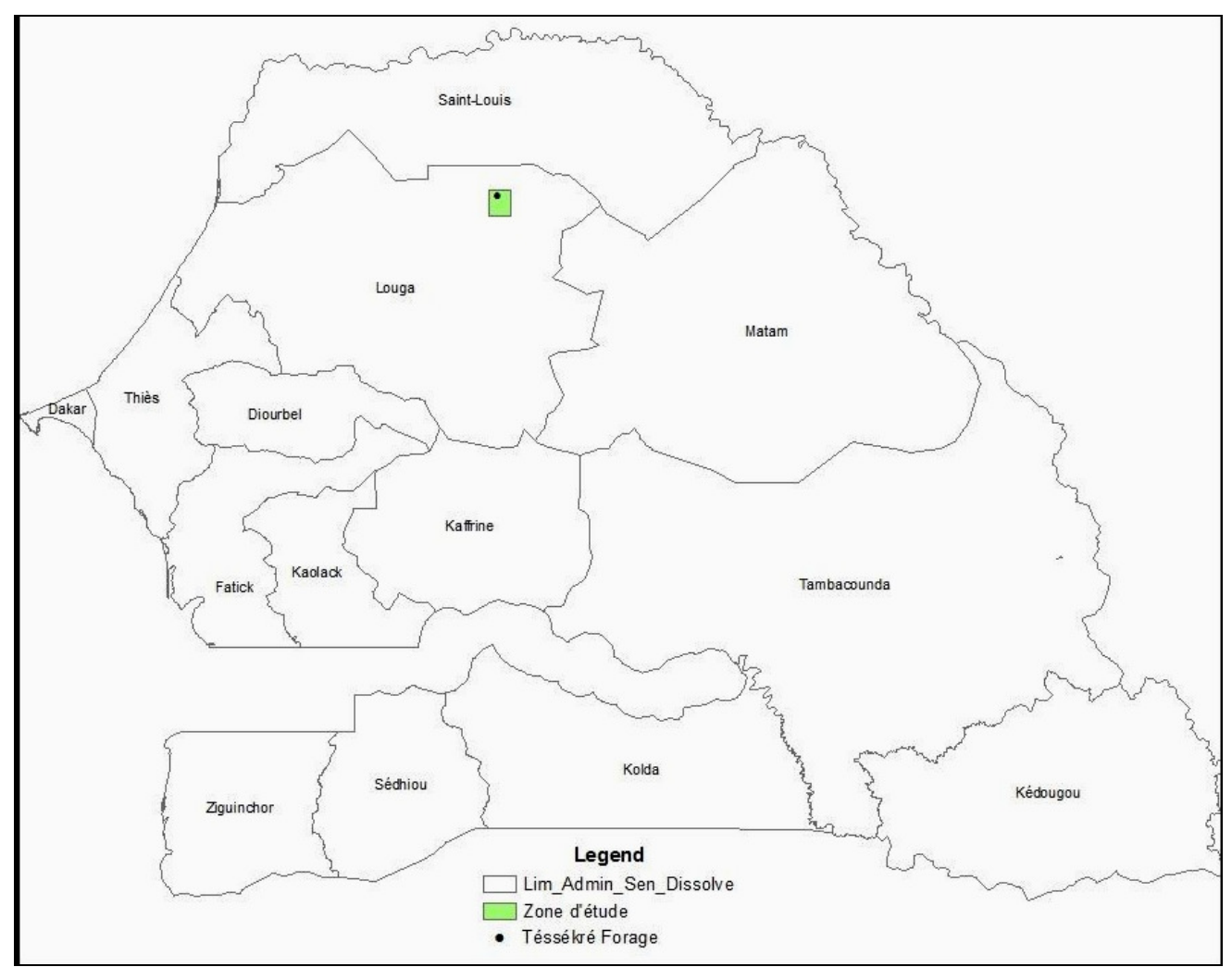

Figure1 : Carte de localisation de Widou Thiengoly de la commune de Téssékéré, région de Louga

Dans le cas où l'informateur est trop âgé ou occupé après l'entretien, nous nous sommes appuyés sur les connaissances du guide pour récolter des spécimens et, un second passage est effectué chez l'informateur pour une vérification des récoltes. Le plus souvent, l'identification des noms botaniques des espèces a été faite sur place à l'aide de la flore du Sénégal (Berhaut, 1967), d'autres ouvrages de références (Adam, 1970, Baumer, 1995; Arbonnier, 2000) et confirmé en

\section{RESULTATS ET DISCUSSION}

Nous avons inventorié 74 espèces réparties dans 32 familles botaniques (Tableau 1). Les familles les plus représentées sont les Leguminoseae $(27 \%)$, les Combretaceae (10\%), les Euphorbiaceae $(7 \%)$, les Rubiaceae (6\%), les Menispermaceae, les Capparaceae et les Anacardiaceae ( $4 \%$ chacune)

Indications thérapeutiques : Les plantes jouent un rôle important dans les problèmes de santé publique. Un peu plus d'une soixantaine de pathologies sont traitées par les espèces médicinales (Tableau1). Ainsi, 22 espèces médicinales sont indiquées pour traiter les affections dermatologiques, 16 contre la tension, 12 comme comparaison avec des échantillons d'herbier de la collection de l'IFAN. La cohérence des informations est vérifiée selon la technique de confrontation des données de El Rhaffari et al. (2002). Une information est considérée comme cohérente lorsqu'elle est rapportée au moins deux fois dans deux localités différentes et par des informateurs différents, si non elle est dite divergente. Seules les informations cohérentes ont été retenues.

revigorantes, 11 contre les maux de ventre, 10 contre la Fatigue, 13 contre les hémorroïdes, 12 contre les parasitoses, 9 contre le diabète. Les autres pathologies sont traitées par 8 espèces au plus. Ces pathologies sont traitées en faisant une décoction, une macération, une infusion, une application externe par massage par exemple ou par malaxation des organes des plantes médicinales. Le plus souvent, les plantes sont utilisées en association. Les bergers soutiennent que chaque espèce a 7 vertus médicinales et 7 autres médicaux- magiques. 
Cisse et al. J. Appl. Biosci. 2016 Ethnobotanique des plantes médicinales chez les bergers peuls de Widou Thiengoly de la commune de Téssékéré (Ferlo-Nord Sénégal).

Tableau1 : Liste des espèces utilisées par les bergers peuls

\begin{tabular}{|c|c|c|c|}
\hline Taxons & Familles & Organes & Indications thérapeutiques \\
\hline Lannea acida A. Rich. & Anacardiaceae & Racine & $\begin{array}{l}\text { Anémie, affections dermatologiques, } \\
\text { problèmes gastriques, hypotension, } \\
\text { revigorant ; médicaux-magique }\end{array}$ \\
\hline Mangifera indica $L$. & Anacardiaceae & Feuille & Tension, Tétanos \\
\hline Sclerocarya birrea (A.Rich.) Hochst. & Anacardiaceae & Écorce & $\begin{array}{l}\text { Diabète, affections dermatologiques, } \\
\text { Prostate, hypertension }\end{array}$ \\
\hline Annona senegalensis Pers. & Annonaceae & Racine & Virilité, Circulation sanguine \\
\hline Annona muricata $L$. & Annonaceae & Feuille & insomnie \\
\hline Calotropis procera (Aiton) W.T.Aiton & Apocynaceae & Racine & Abcès, lutte contre les malfaiteurs \\
\hline $\begin{array}{l}\text { Holarrhena floribunda (G.Don) } \\
\text { T.Durand \& Schinz }\end{array}$ & Apocynaceae & Feuille & Diabète \\
\hline Leptadenia hastata (Pers.) Decne. & Asclepiadaceae & Feuille & $\begin{array}{l}\text { Diabète, affections dermatologiques, } \\
\text { Hémorroïdes, Cancer, Paludisme, Fortifiant, } \\
\text { Circulation sanguine, bourdonnements } \\
\text { d'oreille, idiopathiques }\end{array}$ \\
\hline Asparagus Pauli-guilelmi Solms & Asparagaceae & Tige & antidiurétique \\
\hline Balanites aegyptiaca (L.) Delile & Balanitaceae & $\begin{array}{l}\text { Racine } \\
\text { Fruit }\end{array}$ & $\begin{array}{l}\text { Perte blanche, tension, rhume, maux } \\
\text { de dents, maux de ventre, affections } \\
\text { dermatologiques }\end{array}$ \\
\hline $\begin{array}{l}\text { Newbouldia laevis (P.Beauv.) Seem. ex } \\
\text { Bureau }\end{array}$ & Bignoniaceae & & Appétant, goutte \\
\hline Stereospermum kunthianum Cham. & Bignoniaceae & Racine & $\begin{array}{l}\text { Affections dermatologiques, facilite } \\
\text { lla miction }\end{array}$ \\
\hline Adansonia digitata $L$. & Bombacaceae & Feuille & Abaisse la tension \\
\hline Senna italica (Mill.) Spreng. & Caesalpiniaceae & Feuille & $\begin{array}{l}\text { Constipation, fatigue, Maux de } \\
\text { ventre, laxatif }\end{array}$ \\
\hline Senna occidentalis $L$. & Caesalpiniaceae & Racine & $\begin{array}{l}\text { Virilité, Avortement, Asthme, } \\
\text { Hémorroïdes, affections dermatologiques, } \\
\text { paludisme, Hernie, maux de ventre, } \\
\text { Rétablissement des règles }\end{array}$ \\
\hline Cassia sieberiana DC. & Caesalpiniaceae & Racine & $\begin{array}{l}\text { Parasitoses, Maux de ventre, } \\
\text { Hypoglycémiant, affections dermatologiques, } \\
\text { antibiotique, anti- fatigue, asthénie sexuelle }\end{array}$ \\
\hline $\begin{array}{l}\text { Cordyla pinnata(Lepr.ex A.Rich.) Milne- } \\
\text { Redh. }\end{array}$ & Caesalpiniaceae & Écorce & $\begin{array}{l}\text { Maux de ventre, vers, problème } \\
\text { cardiaque, Ballonnement, Maux de tête }\end{array}$ \\
\hline Detarium microcarpum Guill.\& Perr. & Caesalpiniaceae & Feuille & $\begin{array}{l}\text { Affections dermatologiques, bouffées } \\
\text { de chaleur (ménopause) }\end{array}$ \\
\hline Dialium guineensis Juss. & Caesalpiniaceae & Feuille & $\begin{array}{l}\text { Rhume, hypertension due à l'excès de } \\
\text { sel, conjonctivité }\end{array}$ \\
\hline Parkinsonia aculeata $L$. & Caesalpiniaceae & Feuille & Fatigue, Maux de ventre \\
\hline Piliostigma reticulatum (DC.) Hochst. & Caesalpiniaceae & Écorce & $\begin{array}{l}\text { Maux de dents, Maux de tête, } \\
\text { Hémorroïdes, Hernie, problèmes } \\
\text { prostatiques }\end{array}$ \\
\hline Tamarindus indica $L$. & Caesalpiniaceae & Feuille & $\begin{array}{l}\text { Diabète, courbature, Entorse, Fatigue, } \\
\text { constipation, Rougeole, Fortifiant, sciatique, } \\
\text { épilepsie, problèmes rénaux, vomissements, } \\
\text { parasitoses (vers) }\end{array}$ \\
\hline
\end{tabular}


Cisse et al. J. Appl. Biosci. 2016 Ethnobotanique des plantes médicinales chez les bergers peuls de Widou Thiengoly de la commune de Téssékéré (Ferlo-Nord Sénégal).

\begin{tabular}{|c|c|c|c|}
\hline Boscia senegalensis (Pers.) Lam. & Capparaceae & Feuille & $\begin{array}{l}\text { hémorroïdes, Constipation, Vers, } \\
\text { Nettoie le ventre, Paludisme, Maux de ventre } \\
\text {; problème de reins }\end{array}$ \\
\hline Capparis tomentosa Lam. & Capparaceae & Racine & $\begin{array}{l}\text { rhumatismes, affections } \\
\text { dermatologiques }\end{array}$ \\
\hline $\begin{array}{l}\text { Crateva adansonii DC. } \\
\text { Subsp.adansonii }\end{array}$ & Capparaceae & Racine & Virilité \\
\hline Gymnosporia senegalensis (Lam.) Loes. & Celastraceae & Feuille & $\begin{array}{l}\text { Problème de dents, drépanocytose, cancers, } \\
\text { anti-fatigue }\end{array}$ \\
\hline Chrysobalanus icaco L.subsp.icaco & $\begin{array}{l}\text { Chrysobalanace } \\
\text { ae }\end{array}$ & Feuille & $\begin{array}{l}\text { Diminution du cholestérol, Tension, } \\
\text { hypertension, amaigrissant (obésité) }\end{array}$ \\
\hline $\begin{array}{l}\text { Cochlospermum tinctorium Perr.ex } \\
\text { A.Rich }\end{array}$ & $\begin{array}{l}\text { Cochlospermac } \\
\text { eae }\end{array}$ & Feuille & $\begin{array}{l}\text { Appétit, Digestion, hémorroïdes, } \\
\text { hépatites, drainage du foie }\end{array}$ \\
\hline Anogeissus leiocarpa (DC.) Guill. \& Perr. & Combretaceae & Racine & $\begin{array}{l}\text { Affections dermatologiques, Maux de } \\
\text { dents }\end{array}$ \\
\hline Combretum aculeatum Vent. & Combretaceae & Feuille & Diabète \\
\hline Combretum glutinosum Perr.ex DC. & Combretaceae & Feuille & $\begin{array}{l}\text { Excès de sel, rhume, Fièvre jaune, } \\
\text { hypertension, maux de ventre }\end{array}$ \\
\hline Combretum micranthum G.Don & Combretaceae & Feuille & $\begin{array}{l}\text { Rhume, diabète, hypertension, } \\
\text { diminution du taux de sel, anémie }\end{array}$ \\
\hline Combretum nigricans Lepr. ex Guill. \& Perr. & Combretaceae & Feuille & Accouchement, Fièvre jaune ; kyste \\
\hline Guiera senegalensis J.F.Gmel & Combretaceae & Feuille & $\begin{array}{l}\text { Rhume, Toux, manque d'appétit, } \\
\text { poitrine }\end{array}$ \\
\hline Terminalia avicennioides Guill.\&Perr. & Combretaceae & $\begin{array}{l}\text { Racine } \\
\text { Écorce }\end{array}$ & $\begin{array}{l}\text { Diabète, affectons dermatologiques, } \\
\text { Vision, Hypertension, antidiurétique, Fièvre } \\
\text { jaune, Hémorroïdes }\end{array}$ \\
\hline Ipomoea asarifolia (Desr.) Rome. \& Schult. & Convolvulaceae & Racine & $\begin{array}{l}\text { Affections dermatologiques, } \\
\text { Hémorroïdes }\end{array}$ \\
\hline Merremia aegyptia (L.) Urb. & Convolvulaceae & Racine & Hémorroïdes \\
\hline Euphorbia basalmifera Aiton & Euphorbiaceae & Feuille & $\begin{array}{l}\text { Affections dermatologiques, Vers, } \\
\text { Gonococcie, Maux de dents, Hémorroïdes, } \\
\text { Virilité, Diabète, Après accouchement }\end{array}$ \\
\hline Euphorbia hirta L. & Euphorbiaceae & Feuille & Maux de ventre, dysenterie \\
\hline Bridelia micrantha (Hochst.) Baill. & Euphorbiaceae & Racine & $\begin{array}{l}\text { constipation, problèmes } \\
\text { gynécologiques }\end{array}$ \\
\hline Hymenocardia acida Tul. & Euphorbiaceae & Feuille & Virilité, excès de sel \\
\hline Jatropha chevalieri Beille & Euphorbiaceae & Racine & $\begin{array}{l}\text { Affections dermatologiques, } \\
\text { problèmes prostatiques, aménorrhée } \\
\text { (absence de menstruations) }\end{array}$ \\
\hline $\begin{array}{l}\text { Icacina oliviliformis (Poir.) J.Raynal } \\
\text { var. oliviliformis }\end{array}$ & Icacinaceae & $\begin{array}{l}\text { Feuille } \\
\text { Racine }\end{array}$ & Tache noir \\
\hline Ekebergia capensis Sparrm & Meliaceae & Racine & Maux de ventre \\
\hline Khaya senegalensis (Des.) A.Juss. & Meliaceae & Écorce & $\begin{array}{l}\text { Affections dermatologiques, } \\
\text { Parasitoses, Diabète, Maux de ventre, }\end{array}$ \\
\hline Cissampelos mucronata A. Rich. & Menispermacea & Racine & $\begin{array}{l}\text { Règle douloureuse, affections } \\
\text { dermatologiques }\end{array}$ \\
\hline $\begin{array}{l}\text { Cocculus pendulus (J.R.Forst. \& G.Forst.) } \\
\text { Diels }\end{array}$ & Menispermacea & Racine & Parasitoses, Maux de ventre, Virilité \\
\hline Tinospora bakis (A.Rich.) Miers & Menispermacea & Racine & Fièvre jaune, Paludisme, hépatites \\
\hline
\end{tabular}


Cisse et al. J. Appl. Biosci. 2016 Ethnobotanique des plantes médicinales chez les bergers peuls de Widou Thiengoly de la commune de Téssékéré (Ferlo-Nord Sénégal).

\begin{tabular}{|c|c|c|c|}
\hline & $e$ & & \\
\hline $\begin{array}{l}\text { Acacia nilotica subsp.adstringens } \\
\text { (Schumach. \& Thonn.) Roberty }\end{array}$ & Mimosaceae & Racine & $\begin{array}{l}\text { Ulcère, Maux de dents, affections } \\
\text { dermatologiques, Fatigue, Plaie, } \\
\text { hémorroïdes, Virilité, Hernie, Maux de } \\
\text { ventre, antibiotique, problème de vision, } \\
\text { indigestion ; revigorant }\end{array}$ \\
\hline Acacia seyal Delile & Mimosaceae & Écorce & Affections dermatologiques \\
\hline Entada africana Guill. \& Perr. & Mimosaceae & Racine & Gonococcie \\
\hline Faidherbia albida (Delile) A.Chev. & Mimosaceae & Feuille & $\begin{array}{l}\text { Tuberculose, affections } \\
\text { dermatologiques, Abaisse la tension }\end{array}$ \\
\hline Parkia biglobosa (Jacq.) R.Br.ex G.Don & Mimosaceae & Feuille & Hémorroïdes, Tension \\
\hline Prosopis africana (Guill. \& Perr.) Taub. & Mimosaceae & Écorce & $\begin{array}{l}\text { Anémie, Problème cardiaque } \\
\text { (régulateur cardiaque), Fatigue } \\
\text { générale }\end{array}$ \\
\hline Moringa oleifera Lam. & Moringaceae & Feuille & Diabète, Infection, mauvaise haleine \\
\hline Ximenia americana $L$. & Olacaceae & Feuille & Maux de dents, Maux de tête, asthme \\
\hline $\begin{array}{l}\text { Adenium obesum (Forsk) Roem. et } \\
\text { Schult. }\end{array}$ & Orchideae & Ecorce & $\begin{array}{l}\text { Médicaux-magique, Contre les mauvais } \\
\text { esprits }\end{array}$ \\
\hline Dalbergia melanoxylon Guill. \& Perr. & Papilionaceae & Racine & Médicaux-magique \\
\hline Flemingia faginea (Guill. \& Perr.)Baker & Papilionaceae & Feuille & Hypertension, Excès de sel \\
\hline Pericopsis laxiflora (Benth.) Meeuwen & Papilionaceae & Racine & Rhumatismes \\
\hline Pterocarpus erinaceus Poir. & Papilionaceae & Feuille & $\begin{array}{l}\text { Fatigue, Rhume, Anémie, } \\
\text { hémorroïdes sanglantes }\end{array}$ \\
\hline Tephrosia purpurea (L.) Pers. & Papilionaceae & Feuille & Problème des règles \\
\hline Oxytenanthera abyssinica (A.Rich.) Munro & Poaceae & Feuille & Tension, Asthme, Diabète \\
\hline Ziziphus mauritiana Lam. & Rhamnaceae & Feuille & $\begin{array}{l}\text { hypoglycémiant, Tension, affections } \\
\text { dermatologiques }\end{array}$ \\
\hline Ziziphus mucronata Willd. & Rhamnaceae & Racine & $\begin{array}{l}\text { Antidiurétique, affections } \\
\text { dermatologiques, Virilité }\end{array}$ \\
\hline Gardenia ternifolia Schumach. \& Thonn. & Rubiaceae & Feuille & Fièvre jaune, Tension \\
\hline Mitracarpus hirtus (L.) DC. & Rubiaceae & & $\begin{array}{l}\text { Affections dermatologiques } \\
\text { (traitement externe) }\end{array}$ \\
\hline Mitragyna inermis (Willd.) K.Schum. & Rubiaceae & Écorce & $\begin{array}{l}\text { Infections, Grossesse (accouchement } \\
\text { facile), Arrêt des règles, affections } \\
\text { dermatologiques (traitement interne), } \\
\text { problèmes gynécologiques }\end{array}$ \\
\hline $\begin{array}{l}\text { Sarcocephalus latifolius (Sm.) } \\
\text { E.A.Bruce }\end{array}$ & Rubiaceae & Écorce & $\begin{array}{l}\text { Maux de ventre, Paludisme } \\
\text { (prévention), migraines, Parasitoses, pour } \\
\text { grossir, Constipation, }\end{array}$ \\
\hline Salvadora persica $L$. & Salvadoraceae & Feuille & Asthme, hypertension \\
\hline $\begin{array}{l}\text { Lepisanthes senegalensis (Juss.ex } \\
\text { Poir.) Leenh }\end{array}$ & Sapindaceae & Feuille & $\begin{array}{l}\text { Fatigue, analgésique, Problème de } \\
\text { coagulation, Hémorroïdes, parasitoses }\end{array}$ \\
\hline Sterculia setigera Delile & Sterculiaceae & Écorce & $\begin{array}{l}\text { Tension, antidiurétique, Maux de tête, } \\
\text { Fortifiant, bouffées de chaleur (ménopause) }\end{array}$ \\
\hline Waltheria indica $L$. & Sterculiaceae & Racine & $\begin{array}{l}\text { Virilité, Maux de ventre ; problème } \\
\text { cardiaque }\end{array}$ \\
\hline Zingiber officinale Roscoe & Zingiberaceae & Fruit & Ballonnement de ventre, Virilité \\
\hline
\end{tabular}



Thiengoly de la commune de Téssékéré (Ferlo-Nord Sénégal).

Les parties utilisées: Tous les organes des plantes sont exploités pour traiter les pathologies. Les feuilles $(48 \%)$ sont les organes les plus fréquemment exploités, suivies des racines $(34 \%)$ et des écorces (14\%). Les fruits et les tiges sont peu utilisés dans le traitement des pathologies.

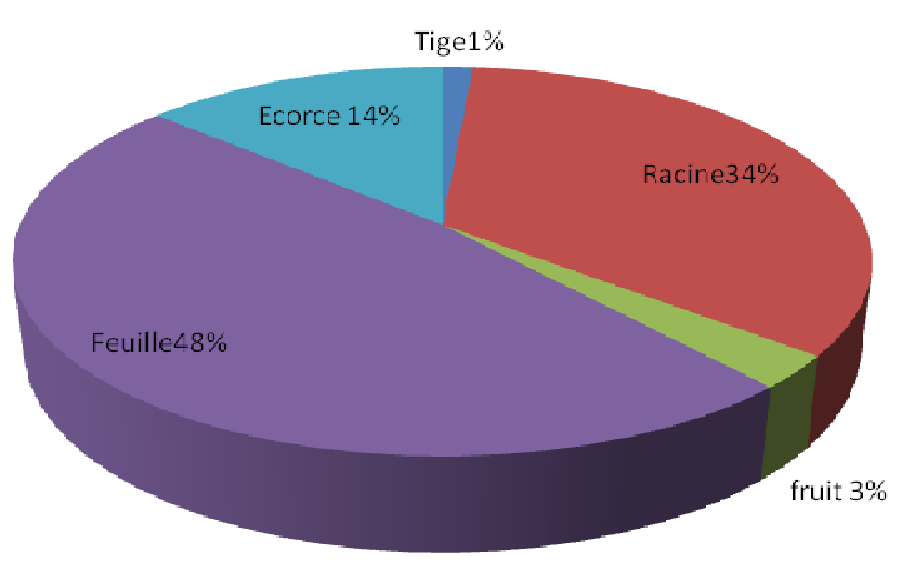

Figure 2 : Organes des plantes exploitées

\section{DISCUSSION}

Les plantes jouent un rôle important dans les problèmes de santé publique. Ainsi, 74 espèces sont citées par les bergers peuls de Widou pour le traitement de certaines pathologies. Toutes les espèces recensées sont bien connues dans la pharmacopée sénégalaise et sont utilisées dans le traitement d'autres pathologies (Kerharo et Adam, 1974 ; Guèye et Diouf, 2007). Si aujourd'hui la médecine traditionnelle suscite autant d'enthousiasme et un renouveau certes incontestable, c'est certainement à cause du rôle très important et à la fois contesté qu'elle joue dans l'amélioration de la santé primaire de la grande majorité des populations des pays du tiers monde (Cissé et al. 2010). En effet, parmi les cinq approches identifiées par Farnsworth (1966) dans la sélection des plantes pour leurs propriétés pharmacologiques, Khafagi et Dewedar (2000) ont montré que l'approche ethnobotanique fournissait les meilleurs résultats dans la découverte de substances bioactives utilisables en médecine. Les feuilles et les

\section{CONCLUSION}

Nous avons inventorié 74 espèces réparties dans 32 familles botaniques. On retrouve une diversité d'espèces dans le traitement d'une pathologie. Cette étude montre combien les ressources végétales jouent un rôle important dans la santé des populations surtout rurales, et, pose en même temps la nécessité de la prise en compte des connaissances ancestrales et traditionnelles racines sont les plus utilisées dans le traitement des pathologies. Ce résultat recoupe celui établi par Giday et al. (2003), Wondimu et al. (2007), Guèye et al. (2012) qui ont indiqué dans leur étude que les feuilles et les racines sont les organes les plus utilisés dans le traitement des pathologies. Ainsi, 12 espèces protégées par le code forestier du Sénégal de 1998, sont utilisées. Ce qui pose un problème de réglementation forestière. Sachant qu'une espèce peut être protégée au Sénégal et contrairement dans la sous région, cette réglementation forestière doit être révisée en étroite collaboration avec les populations locales et les pays de la sous région. La commercialisation des plantes médicinales a été proposée comme moyen pour encourager leur conservation et il est soutenu que, si les populations locales peuvent tirer un revenu de ces espèces végétales, elles seront alors encouragées à les protéger.

dans les politiques de santé publique notamment en Afrique et surtout en zone rurale. En effet, au cours de nos enquêtes il est clairement apparu que les tradipraticiens et les herboristes contribuent grandement à la résolution des problèmes majeurs de santé. Pour une sauvegarde et une valorisation de ces connaissances ancestrales et traditionnelles, nous poursuivons nos 


\section{Cisse et al. J. Appl. Biosci. 2016 Ethnobotanique des plantes médicinales chez les bergers peuls de Widou}

Thiengoly de la commune de Téssékéré (Ferlo-Nord Sénégal).

recherches auprès des populations et tentons en REMERCIEMENTS

Nous remercions l'ensemble de nos informateurs et les bergers peuls de Widou de la commune de Téssékéré (Ferlo-Nord Sénégal). Merci également à l'Unité Mixte

\section{REFERENCES}

Adam JG, 1970. Noms vernaculaire de plantes du Sénégal. Journal d'Agric. Tropic. et de Botanique appliquée, T. XVII, N 7-8-9, JuilletSept. 1970. 112p.

Arbonnier M, 2000. Arbres, arbustes et lianes des zones d'Afrique de l'Ouest. CIRAD (Centre de Coopération Internationale en recherche Agronomique pour le Développement) ; MNHN (Muséum National d'Histoire Naturelle); UICN (Union Mondiale pour la Nature), 544p.

Baumer M, 1995. Arbres et arbrisseaux nourriciers en Afrique occidentale; Enda tiers-monde, Dakar. 210-212p.

Berhaut J, 1967. Flore du Sénégal. Clairafrique, Dakar, $2^{\text {ème }}$ édition, $485 p$.

Cissé A, Guèye M, Koma S, Akpo LE, 2010. Commercialisation des plantes utilisées contre les affections dermatologiques dans les marchés de Dakar et de sa banlieue. Colloque international et interdisciplinaire sur les plantes alimentaires, médicinales et cosmétiques en zone sahélienne. Du 20 au 22 Octobre 2010 Dakar-UCAD.

Code Forestier du Sénégal, 1998. Loi n`98-164 du 20 Février 1998 : Décret d'application. Ministère de l'environnement et de la protection de la nature (MEPN), 10p.

Cunningham AB, 1993. African medicinal plants : Setting priorities at the interface between conservation and primary healthcare. People and Plants Working Paper. UNESCO

DEFCCS (Direction des Eaux et Forêt, Chasses et de la Conservation des sols), 2004. - Rapport national à la $5^{\text {ème }}$ session du forum des Nations Unies sur les forêts. Dakar, Sénégal, Octobre 2004, 24p. (Version PDF).

El Rhaffari L, Zaid A; Hammani K. \& Benlyas M, 2002. Traitement de la leishmaniose cutanée par la phytothérapie au Tafilalet. Revue Biologie \& Santé, Vol. $1, n^{\circ} 4$

Giday M, Asfaw Z, Elmqvist T.and Woldu Z, 2003. An ethnobotanical study of medicinal plants used même temps une caractérisation pharmacologique.

Internationale ESS 3189, à l'Observatoire HommesMilieux Téssékéré (Labex DRIIHM) et à l'institut Klorane qui ont financé nos activités.

by the Zay People in Ethiopia. J. Ethnopharmacol., 85: 43-52.

Guèye M, Diouf M. 2007. Traditional leafy vegetables in Senegal : diversity and médicinal uses. African Journal of traditional Complementary and Alternative Medicine, 4(4) : 469 - 475.

Guèye $M$, Cissé $A$, Diatta $C h D$, Diop $S$, Koma $S$, 2012. Ethnobotanique des plantes utilisées contre la constipation chez les Malinké de la communauté rurale de Tomboronkoto, Kédougou (Sénégal). International Journal of Biological and Chemical Sciences. 9p.

Kerharo J, Adam JG, 1974. La pharmacopée Sénégalaise Traditionnelle: plantes médicinales et toxiques. Ed. Vigot frères, $1011 \mathrm{p}$.

Khafagi IK. and Dewedar A, 2000. The efficiency of random versus ethno-directed research in the evaluation of Sinai medicinal plants for bioactive compounds. J. Ethnopharm. 71 (3): 365-376.

Kilahama FB, 1998. Connaissances écologiques des populations autochtones : outil essentiel des stratégies de vulgarisation rurale. Echos $D u$ Cota, 78 : 14-19.

Martin GJ, 199). Ethnobotany. A method manual. London : Chapman \& Hall, 268p.

MEPN (Ministère de l'Environnement et de la Protection de la Nature), 1998. Plan d'Action Forestier du Sénégal (version révisée).

Okafor JC. et Ham R, 1999. Identification, utilisation et conservation des plantes médicinales dans le sud-est du Nigeria. Thèmes de la biodiversité africaine, №. 3. (8 pp., 1999, anglais/français).

Sofowora EA, 1982. Medicinal plants and traditional medicine in Africa, 2nd Ed., John Wiley \& Sons.

Tabuti JRS, Lye KA. \& Dhillion SS, 2003. Traditional herbal drugs of Bulamogi, Uganda: plants, use and administration. J. Ethnopharmacology, 88, 19-44.

Wondimu T, Asfaw Z. and Kelbessa E, 2007. Ethnobotanical study of medicinal plants around 'Dheera' town, Arsi zone, Ethiopia. J. Ethnopharmacol., 112: 152-161 